\title{
Gute Perspektiven
}

Mit viel Schwung ist die Keramikindustrie ins neue Jahr gestartet. Bestes Beispiel war die Messe ceramitec 2018 vom 10. Bis 13. April, die mehr als 600 Aussteller aus knapp 40 Ländern nach München zog. Präsentiert wurden die neuesten Technologien, Anlagen und Anwendungen, Werkstoffe und Grundstoffe. Die Teilnehmer aus der Keramik- und Pulvermetallurgiebranche zeigten sich investitionsfreudig und in guter Stimmung.

Die neue Sonderschau und ein Vortragsprogramm zur Additiven Fertigung wurden sehr gut angenommen, hieß es beim Messeveranstalter. Besonders das Thema Digitalisierung bzw. Industrie 4.0 war in aller Munde. Im Bereich der technischen Keramik ist man hier schon relativ weit. Neue Möglichkeiten, komplexe Formen in kleinen Stückzahlen kostengünstig und von höchster Qualität zu produzieren, sind vielversprechend.

Die Additive Fertigung könnte die Keramikherstellung revolutionieren, heißt es in der Branche. Sowohl Drucker als auch innovative Werkstoffe wurden dafür vorgestellt. Beispielsweise lassen sich Nanopartikel aus Metall und Keramik inzwischen per 3-D-Druck produzieren. Auch Materialmixe werden neue Anwendungen möglich machen, $z$. B. Kombinationen aus Keramik-Metall oder Keramik-Kunststoff.

Der Anlagenbau zeigte sich ebenso innovativ, nicht nur im 3-D-Druck.
Denn die Fertigung von Hochleistungskeramiken stellen entsprechende Anforderungen auch an Maschinen und Öfen. So waren die Steuerung, aber auch die Simulation von Brennprozessen vielbeachtete Themen.

Ihren Messebesuch können Informationshungrige übrigens schon bald fortsetzen: Die Tecnargilla findet vom 24. Bis 28. September im italienischen Rimini statt. Diese Messe präsentiert ebenfalls auf internationalem Niveau - mit Unternehmen aus den Bereichen technische Keramik, Feuerfestwerkstoffe und keramische Bauprodukte wie Fliesen, Ziegel, Steine, aber auch Sanitärprodukte und Geschirr. In diesem Jahr wollen sich die Veranstalter auch den ästhetischen Trends bei Keramikprodukten widmen; außerdem wurde das Thema GreenTech neu aufgenommen. Denn Zeit, Energie und Rohstoffe zu sparen oder Emissionen und Abfall gemäß EU-Vorgaben zu vermeiden (Stichwort Kreislaufwirtschaft), sind Anliegen, die auch für die Keramikindustrie zunehmend in den Vordergrund rücken.

Wie auf der ceramitec, wird auch auf der Tecnargilla das Team der beiden Zeitschriften INTERCERAM und Keramische Zeitschrift aus dem Hause Springer Fachmedien Wiesbaden $\mathrm{GmbH}$ vor Ort sein. Wir berichten und laden Sie ein zur Kontaktaufnahme.

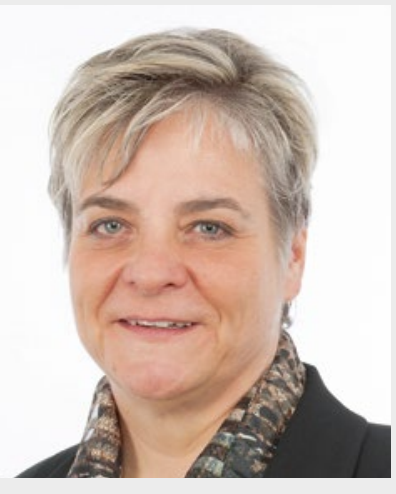

Dr. Wiebke Sanders Verantwortliche Redakteurin wiebke.sanders@springernature.com 